\title{
A placebo-controlled study to investigate the effect of Dog Appeasing Pheromone and other environmental and management factors on the reports of disturbance and house soiling during the night in recently adopted puppies (Canis familiaris) ${ }^{\text {is }}$
}

\author{
Katy Taylor, Daniel S. Mills* \\ Animal Behaviour, Cognition and Welfare Group, University of Lincoln, \\ Department of Biological Sciences, Riseholme Park, Lincoln LN2 2LG, UK
}

Available online 29 November 2006

\begin{abstract}
Disturbance and house soiling during the night are common problems faced by the new puppy dog owner. They may result as consequence of a mismatch between the developmental status of the puppy and its new environment and/or separation distress in a typically social animal. The aim of this study was to examine the effect of Dog Appeasing Pheromone (DAP, Ceva Santé Animale) as well as a range of other management and environmental factors that might affect this process. It has been suggested that DAP may help the puppy settle into the new home by continuing the provision of the maternal appeasing pheromone. In order to test this, a double-blind, placebo-controlled trial of DAP was conducted with 60 pedigree puppies, aged between 6 and 10 weeks, as they entered their new home. A few days prior to the puppy's arrival, volunteer owners were supplied with either a verum or placebo plug-in diffuser designed for the slow release of the pheromone analogue over 4 weeks. Owners reported daily on disturbance and house soiling during the previous night over the following 8 weeks from the puppy's first night in the new home. The effect of the treatment together with the gender of the puppy, its maternal environment, the use of a puppy crate, sleeping with other dogs at night and the experience of the owner were included in a general linear model to explain the total number of nights spent disturbing and house soiling. Sleeping with another dog reduced the puppies' tendency to disturb at night to almost zero. Over $70 \%$ of puppies sleeping alone disturbed during the first night. The mean total number of nights of disturbance over the 2 months was between five and six
\end{abstract}

\footnotetext{
This paper is part of the special issue entitled "Veterinary Behavioural Medicine" guest edited by Daniel Mills and Gary Landsberg.

* Corresponding author. Tel.: +44 1522 895356; fax: +44 1522895328.

E-mail address: dmills@lincoln.ac.uk (D.S. Mills).
} 
nights, mostly in the first week in the home. A significant effect of DAP treatment was found in the case of the gundog breeds only ( $p=0.003$ ), gundogs receiving placebo cried for a median of nine nights, those receiving verum cried for a median of three nights. No effect of DAP treatment was observed on the total number of nights that the puppy soiled the house $(p>0.05)$. However, puppies that were placed in crates during the night $(p=0.004)$ or had come from domestic maternal environments $(p=0.006)$ had significantly fewer reports of house soiling over the first 2 months in the new home.

(C) 2006 Elsevier B.V. All rights reserved.

Keywords: Crate; Dog; House soiling; Maternal environment; Pheromone; Puppy; Separation distress; Vocalisation

\section{Introduction}

It is widely recognised that entry into a new home is a stressful time for a new pet dog. For youngsters it may coincide with removal of the mother and littermates as well as transferral from a familiar location to a completely novel one (Elliot and Scott, 1961; Pettijohn et al., 1977; Slabbert and Rasa, 1993; Serpell and Jagoe, 1995). In particular, being placed in isolation, whether this is from conspecifics or other species including humans, may result in separation distress which is typified by high frequency vocalisations and escape attempts (Elliot and Scott, 1961; Cairns and Werboff, 1967; Cohen and Fox, 1976; Pettijohn et al., 1977; Gurski et al., 1979). Separation protest is an adaptive behaviour in puppies since it phylogentically serves to help reunite the puppy with its mother or other conspecifics thus maximising its safety (Cohen and Fox, 1976; Voith and Borchelt, 1996). The tendency to protest vocally when isolated tends to peak at around 6-9 weeks of age (Elliot and Scott, 1961). Unfortunately this coincides with the popular age for adoption of the puppy into the family home. Puppies are usually isolated during the night in the home and, perhaps as a result, disturbance at night primarily by vocalisation is a commonly reported problem for new puppy owners (Askew, 1996). Another challenge for the new puppy owner is housetraining. Soiling in the house by the puppy when unsupervised, such as during the night, may reflect developmental immaturity and/or separation distress (Voith and Borchelt, 1996).

Although typically both disturbance and house soiling during the night resolve over time (Askew, 1996), as the puppy ages and adjusts to its new environment, the average length of time for which the puppy presents with these problems has not been well documented. In addition, a number of factors may explain the incidence and length of time for which puppies present with these behaviours. Scott and Fuller (1965), Gurski et al. (1979) and Pettijohn et al. (1977) reported some differences between breeds in tendency to protest vocally. Bradshaw et al. (2002) reported both breed and gender differences in tendency towards separation anxiety in juvenile dogs. The environment into which the puppy is being placed may also affect its development. For example, the experience of the owner with other dogs may affect their ability to settle and train their new puppy. Separation related and other problems have been associated with first-time owners (Jagoe and Serpell, 1996; Ledger, 2000). Similarly, the presence of other dogs in the home may also provide some continuity in the puppy's environment and in particular prevent disturbance at night. For example, Pettijohn et al. (1977) reported that separation distress could be significantly alleviated by the presence of a conspecific. The environment from which the puppy has just been removed may also have an effect. In a retrospective study, Appleby et al. (2002) found that dogs that were reportedly reared in non-domestic maternal environments, i.e. kennels or outhouses, were 
more likely to present with problems relating to aggression and avoidance behaviour in later life. This may be due to reduced opportunities for socialisation and other learning experiences in these environments. Non-domestic maternal environments may therefore be expected to also have a more immediate effect on the puppy's ability to settle into the new home. Finally, the use of puppy crates may also have an effect on disturbance and house soiling during the night. These are cages or pens that contain the puppy in a relatively small space and are typically used for house training purposes or to limit the effects of puppy chewing or separation anxiety (Voith and Borchelt, 1996). However, their efficacy at actually reducing elimination has not been reported in the scientific literature.

Disturbance and house soiling also impact on the owner in addition to suggesting that the puppy is having difficulty adjusting to its new environment. While they continue, the owner may suffer from disturbance in sleep patterns and inconvenience in addition to anxiety regarding the welfare of the puppy. It is important that such behaviours are resolved rapidly since they can also negatively affect the relationship between dog and owner. Both inappropriate elimination and separation related behaviours are commonly given reasons for relinquishment of the dog to an animal rescue shelter (Patronek, 1996; Wells and Hepper, 2000; Miller et al., 1996). Owners may be tempted to return to the puppy and comfort it when it vocalises which may encourage the problem further (Askew, 1996). The alternative may be to allow the puppy to sleep near the owner. However, perhaps because of perceived difficulties with reversing this behaviour when the puppy is older, and the possibility of its perceived contribution to separation related or aggressive behaviour in the future (Askew, 1996; Jagoe and Serpell, 1996), this may not be widely adopted. Owners report using hot water bottles, soft toys and music to sooth the puppy but, to date, none have been shown to be consistently effective.

Dog Appeasing Pheromone (DAP, Ceva Santé Animale, France) is a synthetic analogue of the maternal appeasing pheromone which is thought to promote reassurance and attachment of the puppy to the mother (Pageat, 1999). It can be delivered into the home via a plug-in air diffuser and has been reported to be useful in relieving canine anxiety associated with firework noises (Sheppard and Mills, 2003), travelling (Gandia Estelles and Mills, 2006), being left alone (Gaultier et al., 2005) and within the rescue shelter environment (Tod et al., 2005). In all these situations, tendency to vocalise in particular was reduced. However, these studies have largely involved adult dogs, but since this pheromone is produced when the bitch is suckling her puppies, it was hypothesised that continued provision in the new home may also help familiarise the environment to the puppy and thus comfort them particularly when they are left alone. This hypothesis was further supported by case studies by the second author (Mills, unpublished data) which have suggested that DAP is useful in helping puppies settle into their new home by reducing the frequency of disturbed nights and maintaining the puppy's emotional stability. However, there have been no controlled studies of the effect of DAP on vocalisation at night by puppies.

Therefore the aim of this study was to evaluate the effects of DAP on disturbance and house soiling at night, taking into account other factors such as gender, breed and differences in previous and current environment. Control for the non-specific effects of treatment was accommodated through the use of a placebo device, and due to the usual resolution of the problem with age, comparison within subjects was not possible. Comparison in the number of reports of house soiling and disturbance was therefore made between two groups of puppies in the form of a longitudinal, randomised, controlled trial. 


\section{Methods}

\subsection{Recruitment of volunteers}

Volunteer participants were sought from members of the public who had placed an order for a puppy with one of a number of pedigree dog breeders in the local area. In order to be suitable for inclusion onto the trial, puppies had to be healthy, still in the possession of the breeder prior to the start of the trial, expected to be between 6 and 10 weeks of age when adopted and going to new homes, i.e. not remaining with the breeder. A few days prior to the puppy's expected arrival in the new home, the owner was visited by the researcher (KT). The method of assessment of the puppy's behaviour was explained, informed consent obtained and a diffuser (containing either placebo or DAP) installed in the room where the puppy was expected to rest during the night. Recruitment onto the trial ceased when 60 owners had joined the trial.

\subsection{Allocation of treatments}

The study was conducted as a double-blind, placebo-controlled, longitudinal study. Sixty diffusers designed for the slow release of DAP into a room over 4 weeks were made available for the study (Ceva Santé Animale, France). Thirty of these diffusers contained DAP (verum) and 30 contained the mineral oil carrier fluid only (placebo). The diffusers were labelled as A or B to enable balancing of the recruited subjects with respect to age at adoption, breed group and the presence of another dog in the home, after the first 30 subjects had been allocated by random number generation. Recruits from the same litter were difficult to obtain, but where this did occur, supply of diffusers was also balanced. The identity of A and B was not released to the researcher, trial supervisor or recruits until all the statistical analyses had been completed.

\subsection{Assessment of disturbance and house soiling at night}

Puppies were monitored for the purposes of the trial for a total of 8 weeks following their adoption. Owners were requested to indicate on a personalised calendar whether during the night the puppy had: (1) 'disturbed them' and (2) 'gone to the toilet'. Owners were instructed that disturbance included primarily vocalisation but also persistent scratching at the door and that going to the toilet included urination and defecation. 'During the night' included the time from being left alone for the night to the time in the morning the owner would normally intend to rise. They were instructed to complete the form the following morning of each night. Contact by telephone was made by the same researcher during the first, fourth and eighth week after adoption in order to check compliance.

\subsection{Other independent variables}

A range of details regarding each puppy were also recorded for the purposes of explaining the variation in disturbance and house soiling. These are listed in Table 1 together with the numbers of puppies revealed to be in each category.

\subsection{Statistical analyses}

A regression equation was fitted to the proportion of puppies reported to house soil and disturb each of the first 56 nights in the new home. As an exploratory analysis, a general linear model (GLM; Minitab v13.3, Minitab, Inc., USA 2000) was fitted to firstly, the total number of nights the puppy was reported to disturb the owner, and secondly, the total number of nights the puppy was reported to soil the house, during the night over the 8-week period. For each model, the fixed effect of treatment and the random effects of sex, breed (gundog or other), use of puppy crate, owner's experience and maternal environment were included as explanatory factors. Each factor therefore had one degree of freedom and an approximately equal proportion 
Table 1

Factors included in the general linear model to explain disturbance and house soiling, with the number of puppies each category $(N=60)$

\begin{tabular}{ll}
\hline Factor & Group (number of puppies) \\
\hline Treatment & Verum (30), placebo (30) \\
Gender & Female (33), male (27) \\
Breed & Gundogs (25), terriers (16), working (12), hounds (3), utility (3), \\
& toy (1) (British Kennel Club groupings) \\
Use of a puppy crate & Routinely placed into a puppy crate at night (29), free-roaming \\
& within room (31) \\
Owner experience & Owner had, as an adult, previously owned a dog (44), no experience (16) \\
Maternal environment & Non-domestic (35), domestic (25) \\
Presence of another dog at night & Slept alone (49), slept with another dog, not crated apart (11) \\
\hline
\end{tabular}

of puppies in each group. The least significant factors and interactions were serially removed to decrease the degrees of freedom in each model. When all remaining factors in the model had a $p$-value of less than 0.1 , first-order interactions were included to assess any improvement in the model, but were removed if there was none. Post hoc analysis was carried out using a non-parametric, two-sample test on the total number of nights spent house soiling or disturbing between groups as described (Mann-Whitney; Minitab v13.3, Minitab, Inc., USA 2000).

\section{Results}

\subsection{Effect of the presence of another dog}

Following the recruitment of volunteers, it was reported to the authors, that some adult dogs produce an appeasing pheromone similar to DAP from the base of their ears (Pageat, personal communication). As a consequence of this finding, 11 puppies that slept with other dogs were removed from the analysis since they may have been in receipt of additional, uncontrolled pheromone treatment. Post hoc analysis indeed found a significant effect of sleeping with another $\operatorname{dog}$ on reports of disturbance (Mann-Whitney; $N=49$ (without dog), $N=11$ (with $\operatorname{dog}$ ), $W=1691.5, p<0.001$ ). Puppies sleeping with another dog cried a median of zero nights (range 0-3), compared to four (range 0-25) for those sleeping alone. No effect of sleeping with another dog was found for house soiling (Mann-Whitney; $N=49$ (without dog), $N=11$ (with $\operatorname{dog}$ ), $W=397.5, p=0.240)$.

\subsection{Progression of disturbance and house soiling in single puppies}

Puppies were a mean of 8.3 weeks of age at adoption (std. dev. 1.3 weeks) and were typically left alone in a downstairs room at night. Puppies not sleeping with other dogs were reported to

Table 2

The average total number of nights that single puppies were reported to disturb their owners and house soil during the night in the first 8 weeks in the new home $(N=49)$

\begin{tabular}{lccrr}
\hline Reported behaviour & Total mean nights & Std. dev. & Median & Range \\
\hline Disturbance & 5.5 & 5.4 & 4 & $0-25$ \\
House soiling & 23.4 & 17.7 & 21 & $0-56$ \\
\hline
\end{tabular}


disturb their owners for a mean of 5.5 nights over the first 56 nights in the home, however there was a large range to this reported occurrence, see Table 2. Seventy-one percent of puppies sleeping alone were reported to disturb during the first night in the new home irrespective of treatment, see Fig. 1. The proportion of puppies reported to disturb decreased logarithmically during the first week, with $22 \%$ being reported to have disturbed on the seventh night. By the end of the 14th night in the new home less than $15 \%$ of puppies were reported to disturb on any given night and after the 28th night only one or two puppies were reported to disturb intermittently. The line of best fit represented a log-linear regression; $\log _{10}$ (proportion of puppies disturbing $)=0.46-0.03$ night $(R$-squared $=81.3 \%$, d.f. $1,36, p<0.001)$.

Reports of house soiling had a longer time to resolution than disturbance, with puppies soiling on average 23.4 nights during the first 56 nights in the new home, see Table 2. Eight-four percent of puppies were reported to soil their living area during their first night in the new home, see Fig. 1. Decrease in reports of house soiling appeared to be more linear than for disturbance, with $65 \%$ soiling on the 7 th night, $59 \%$ soiling on the 14 th night, $53 \%$ on the 21 st and $45 \%$ on the 28 th night in the new home. Towards the end of the study period ( 8 weeks) $10-20 \%$ of puppies were still being reported to be unable to control their elimination during the night. The line of best fit represented a linear regression; proportion of puppies house soiling $=0.74-0.01$ night $(R$ squared $=94.3 \%$, d.f. $1,54, p<0.001)$.

\subsection{Risk factors for disturbance at night}

Two puppies, one from each treatment group, were reported to disturb for a prolonged period (23 and 25 nights in total, 12 nights more than the next puppy). They were contributing inordinately to the degree of variation in this variable and for this reason were removed from further analysis. After sequential knockout of the least significant factors, the general linear

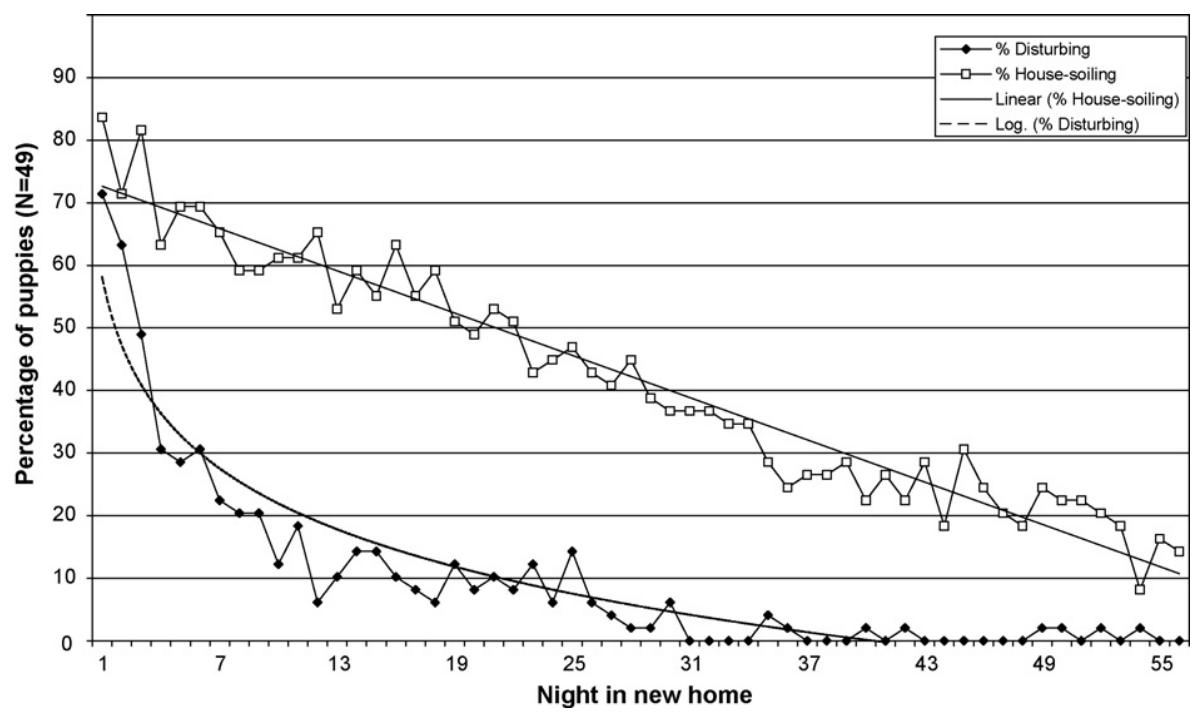

Fig. 1. The percentage of puppies sleeping without another dog that were reported to disturb their owners (filled diamonds) and the percentage reported to soil the house (open squares) during the night, on each night, from the 1 st night to the 56th night following adoption. Regression lines as described in the text are shown. 
Table 3

Factors remaining in the final general linear model analysis of variance affecting the total number of nights that single puppies were reported to disturb their owner during the night over the first 8 weeks in the new home $(N=47)$

\begin{tabular}{llll}
\hline Factor & d.f. & $F$ & $p$-Value \\
\hline Treatment (verum, placebo) & 1,43 & 0.46 & 0.622 \\
Breed (gundog, other) & 1,43 & 0.51 & 0.605 \\
Treatment $\times$ breed interaction & 1,43 & 9.85 & 0.003 \\
\hline
\end{tabular}

model retained the factors 'treatment' and 'breed', with a significant interaction occurring between the two $(p=0.003)$, see Table 3 .

The interaction between treatment and breed was explored post hoc and was found to represent a treatment effect for the largest breed group, namely 'gundogs'. Gundogs in receipt of DAP were reported to disturb for a median of three nights. Gundogs in receipt of placebo were reported to disturb for a median of nine nights in total (Mann-Whitney test; $N=14$ (DAP), $N=7$ (placebo), $W=117.5, p=0.003$ ). There was no significant difference in reported disturbance at night between treatment groups amongst the other breeds $(p>0.05)$, with both groups being reported to disturb for a median of three nights. This result is illustrated in Fig. 2 using the mean total number of disturbed nights.

Since gundogs appear to disturb for longer periods in the absence of DAP, this intervention might therefore prevent prolonged disturbance in those puppies that show a tendency to disturb. To test this hypothesis, the total number of disturbed nights was compared between treatment groups using only those puppies with a tendency to disturb (classified post hoc as those that disturbed on at least two of the first three nights in the new home). A significant effect of treatment was found (Mann-Whitney; $N=15$ (DAP), $N=14$ (placebo), $W=173.5, p=0.025$ ). Irrespective of breed, puppies with a tendency to cry disturbed their owner for a median of three

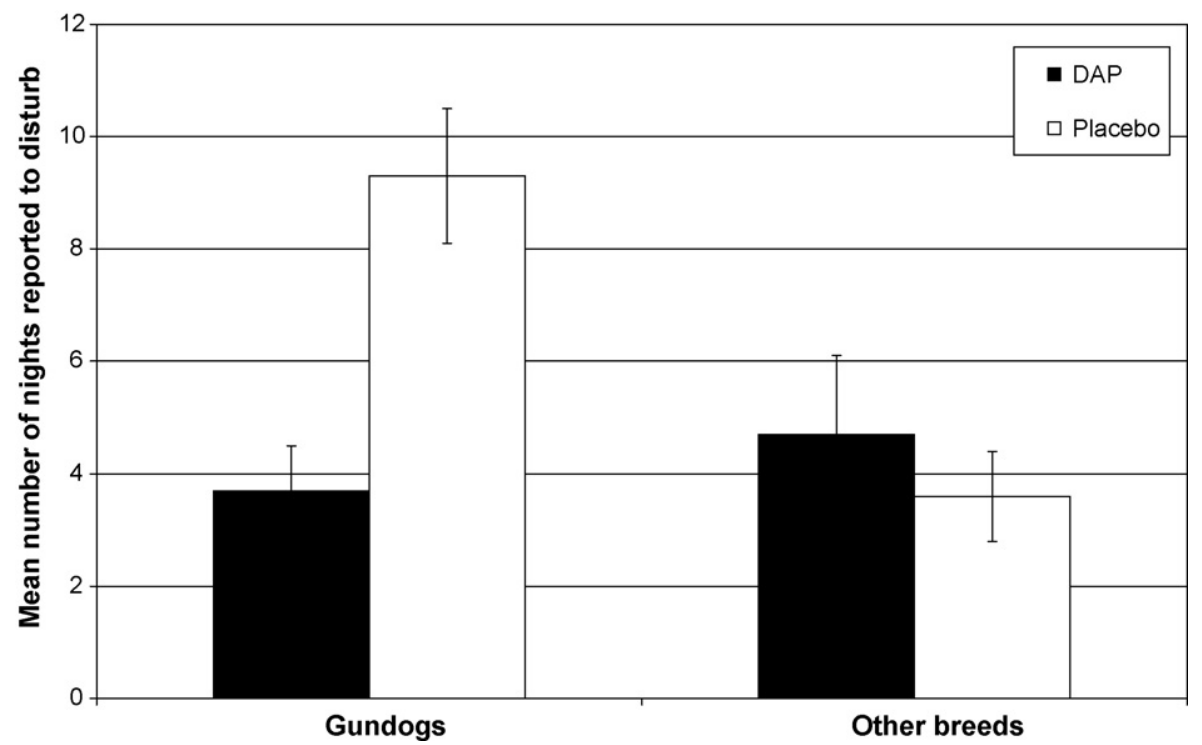

Fig. 2. The mean total number of nights that gundogs (DAP $N=14$, placebo $N=7)$ and other breeds $(\mathrm{DAP} N=9$, placebo $N=17$ ) were reported to disturb at night during the first 8 weeks in the new home (standard error bars are shown). 
Table 4

Factors remaining in the final general linear model analysis of variance affecting the total number of nights that single puppies were reported to soil the house during the night over the first 8 weeks in the new home $(N=49)$

\begin{tabular}{llll}
\hline Factor & d.f. & $F$ & $p$-Value \\
\hline Maternal environment (non-domestic, domestic) & 1,46 & 6.14 & 0.017 \\
Crated at night (yes, no) & 1,46 & 5.80 & 0.020 \\
\hline
\end{tabular}

nights if they were in receipt of DAP. Those with a tendency to cry that were in receipt of placebo cried for a median of seven nights.

\subsection{Risk factors for house soiling at night}

Maternal environment and use of a puppy crate were significant random factors in the final general linear model explaining the total number of nights reported to soil the house, see Table 4. An interaction between these two factors did not improve the model. Puppies that were crated at night were reported to soil their living area for a median total of 10 nights compared to 30 nights if left unrestricted (Mann-Whitney; $N=26$ (crated), $N=23$ (not crated), $W=507.5, p=0.004$ ). The maternal environment the puppy came from had a similar effect on reports of house soiling. Puppies from domestic maternal environments were reported to soil the house a median total of 16 nights compared to 30 for those from non-domestic environments (Mann-Whitney; $N=26$ (non-domestic), $N=23$ (domestic), $W=787.5, p=0.006$ ). There was no significant effect of treatment on total number of nights the puppy was reported to soil the house during the first 8 weeks in the new home $(p>0.05)$.

\section{Discussion}

Disturbance and house soiling during the night in recently adopted puppies are behaviours that appear to reflect a mismatch between the developmental status of the puppy and its new environment. They may or may not also include separation distress on the part of the puppy. In this study both problems were reported in the majority of single puppies during their first night in the new home. Disturbance at night appeared to resolve more rapidly than house soiling, which might suggest that it is an acute response to separation that is less affected by developmental/ training factors. The average puppy continued to disturb for another four or five more nights, mostly during the first week, although a proportion of puppies disturbed for substantially longer. House soiling improved linearly, with nearly $50 \%$ of the puppies still soiling after 1 month. Between 10 and 20\% remained untrained, at least through the night, after the first 2 months in the new home.

The study provided evidence to suggest that DAP can help prevent the tendency for prolonged disturbance at night. However, another study would be required to assess its efficacy as a treatment of the problem in individuals already showing signs. It is hypothesised that the initial introduction into the new home and subsequent isolation at night may be so stressful that any effect of DAP at this time is overwhelmed. Since those puppies in the study that had another dog with which to sleep rarely disturbed their owner, it would seem that physical access to a conspecific at this time may be the most effective buffer of the problem. This may be because they also provide a source of appeasing pheromone to the puppy (Pageat, personal communication) in addition to their physical presence. 
Puppies that were not sleeping with another dog and showed a tendency to disturb at night during the first 3 days in the new home tended not to continue to disturb in the presence of DAP. Those with a tendency to disturb that were not in the presence of DAP tended to continue to disturb on more occasions. The apparent breed effect might have arisen because gundogs were more likely than other breeds to disturb at night for a prolonged period in the absence of DAP, but the effect was strongest when considering breed rather than tendency for prolonged crying. It is believed that the chemical structure of the pheromone itself is not breed-specific (Pageat, 1999) and no other reports of a breed interaction have been published. The breeds representing gundogs in this study included mostly Labrador retrievers, Weimeraners and Spaniels. A recent longitudinal study found that Labrador retrievers were more likely than border collies to be reported with separation related behaviours between 3 and 18 months of age (Bradshaw et al., 2002). Gundog breeds are particularly popular as family pets and selection for increased sociability and ability to recall may have resulted in animals that cope less well to separation, particularly from humans. However, as the study by Bradshaw et al. (2002) found that the Labradors also received more social referencing from their breeders than the collies, this effect may reflect differences in early environment as opposed to genuine genetic tendencies. No gundogs were used in the early studies of Scott and Fuller (1965), Gurski et al. (1979) and Pettijohn et al. (1977) on separation protest so no comparison with their work can be made. The apparent relationship between separation distress and response to treatment in gundogs is worthy of further investigation through another study involving fewer breeds and more dogs.

House soiling during the night was most influenced by prior and current environmental factors, both of which may be related to training. Puppies that were reared in domestic environments (i.e. within the breeder's home) were less likely to be reported to house soil during the night than those from non-domestic environments (i.e. kennels and outhouses). One explanation for this may be that breeders in domestic settings are in a better position to begin early house training, whether this is by conscious effort on their part or more simply, by allowing the puppies to follow the mother outside to eliminate. This finding may therefore be seen to support the suggestion in Appleby et al. (2002) that non-domestic maternal environments provide fewer opportunities for early learning than domestic environments. Appleby et al. (2002) found evidence for a more long term effect of maternal environment on behaviour; this study provides an additional short term effect. The effect of a domestic maternal environment appears to speed up housetraining overnight by an order of two, although house soiling at night may not necessarily be an accurate measure of house training during the day. Independently to this, there also appears to be a similar, slightly larger effect of crating the puppy over night on house soiling. Since owners were asked if the puppy 'went to the toilet' this effect of crating appears to be real and not a reflection of any misunderstanding between elimination and house soiling. The theory behind crating is that the puppy will resist soiling its own bedding and so learns more rapidly to hold its bladder, however, it may also be that crating limits access to water and exercise, both of which may have more impact on the tendency to urinate (Voith and Borchelt, 1996). Although the effect of crating on house training per se cannot be assessed as part of this study it does appear at least to significantly reduce the tendency to eliminate during the night.

No evidence could be found for an effect of gender of puppy on disturbance at night or house soiling. By contrast, the cross-sectional study of Bradshaw et al. (2002) found an association between gender and reports of current separation related behaviour, although their longitudinal study did not. Flannigan and Dodman (2001) also failed to find evidence of a relationship between gender and separation anxiety. The lack of evidence of an effect of the experience of the owner is perhaps surprising since the experience of the owner has been linked to problems in 
adult dogs, including separation related behaviour (Jagoe and Serpell, 1996; Ledger, 2000). However, since most owners had some experience of owning a dog a large discrepancy between groups may be necessary in order to have confidence of observing an effect with this sample size. Voith and Borchelt (1996) also noted that speed of house training may have more to do with the fastidiousness of the owner as a house keeper rather than their experience as a dog owner. Age of the dog is likely to have an effect on both problems, but since most puppies were between 7 and 9 weeks of age it was not included as a factor in this study.

It might be argued that the measures of disturbance and house soiling used in this study were crude in that neither the quality nor quantity of the disturbance or house soiling during each night was recorded. However, these simpler recording methods should be less subject to rater bias and so potentially allow a more accurate assessment of the behaviours that are considered a problem to the owner. The simpler method may have also have contributed to the excellent retention of participants and compliance. Nonetheless, a further study looking more specifically at the volume, frequency, type and duration of the disturbance during each night might provide more information about both breed tendencies and the more subtle effects of interventions.

The relationship between tendency to protest at night when initially adopted and tendency to perform separation related behaviours as a juvenile and adult is not fully understood. A connection between the two may be seen in the observation that the vocalisations associated with separation anxiety in adult dogs resemble the high frequency protest calls of young puppies (Overall et al., 1999). If DAP prevents prolonged disturbance at night at the adoption stage in a puppy's life then it may also do so at other sensitive developmental or environmental periods. It remains to be seen whether there are any other long term effects on behaviour.

\section{Conclusion}

Crying at night is unlikely to occur in the presence of other dogs and so where puppies are going to a home with other dogs they should be allowed to sleep with them, if is safe to do so. Where puppies are going to homes with no other dogs, DAP may help to reduce the likelihood of prolonged disturbance at night, particularly if the puppy is a gundog. Puppies that are kept in puppy crates during the night or have come from domestic maternal environments had fewer reports of house soiling during the night, which may reflect faster housetraining.

\section{Acknowledgements}

The authors would like to thank Ceva Sante Animale, France for support of the first author and provision of the verum and placebo diffusers. The authors would also like to thank the breeders who supplied volunteers and to the puppy owners themselves for their efforts.

\section{References}

Appleby, D.L., Bradshaw, J.W.S., Casey, R.A., 2002. Relationship between aggressive and avoidance behaviour by dogs and their experience in the first six months of life. Vet. Rec. 150, 434-438.

Askew, H.R., 1996. Treatment of Behaviour Problems in Dogs and Cats: A Guide for the Small Animal Veterinarian. Blackwell Science, Oxford, UK, p. 220.

Bradshaw, J.W.S., McPherson, J.A., Casey, R.A., Larter, S., 2002. Aetiology of separation-related behaviour in domestic dogs. Vet. Rec. 151, 43-46.

Cairns, R.B., Werboff, J., 1967. Behaviour development in the dog: an interspecific analysis. Science 158, 1070-1072.

Cohen, J.A., Fox, M.W., 1976. Vocalisation in wild canids and possible effects of domestication. Behav. Process. 1, 77-92. 
Elliot, O., Scott, J.P., 1961. The development of emotional distress reactions to separation in puppies. J. Genet. Psychol. $99,3-22$.

Flannigan, G., Dodman, N.H., 2001. Risk factors and behaviours associated with separation anxiety in dogs. J. Am. Vet. Med. Assoc. 219, 460-466.

Gandia Estelles, M., Mills, D.S., 2006. Signs of travel related problems in dogs and their response to treatment with dog appeasing pheromone. Vet. Rec. 159, 143-148.

Gaultier, E., Bonnafous, L., Bougrat, L., Lafont, C., Pageat, P., 2005. Comparison of the efficacy of a synthetic dogappeasing pheromone with clomipramine for the treatment of separation-related disorders in dogs. Vet. Rec. 156, 533538.

Gurski, J.C., Davis, K., Scott, J.P., 1979. Interaction of separation discomfort with contact comfort and discomfort in the dog. Dev. Psychobiol. 13, 463-467.

Jagoe, A., Serpell, J., 1996. Owner characteristics and interactions and the prevalence of canine behaviour problems. Appl. Anim. Behav. Sci. 47, 31-42.

Ledger, R.A., 2000. Owner and dog characteristics: their effects on the success of the owner-dog relationship. Part 3. Dog owners and decision making: how to predict their behaviour! Vet. Int. 12, 25-31.

Miller, D.D., Staats, S.R., Partlo, C., Rada, K., 1996. Factors associated with the decision to surrender a pet to an animal shelter. J. Am. Vet. Med. Assoc. 209, 738-742.

Overall, K.A., Agulnick, L., Dunham, A.E., Kapes, M., Seksel, K., Frank, D., 1999. Qualitative and quantitative differences in vocalisation by dogs affected with separation anxiety and unaffected dogs using sonographic analysis. In: Proceedings of the Second World Meet. Vet. Ethol., Lyon, September 21-22, pp. 109-115.

Pageat, P., 1999. Attachment and pheromones in the dog. In: Proceedings of the Second World Meet. Vet. Ethol., Lyon, September 21-22, p. 7.

Patronek, G.J., 1996. Risk factors for relinquishment of dogs to an animal shelter. J. Am. Vet. Med. Assoc. 209, 572-581.

Pettijohn, T.F., Wont, T.W., Ebert, P.D., Scott, J.P., 1977. Alleviation of separation distress in 3 breeds of young dogs. Dev. Psychobiol. 10, 373-381.

Scott, J.P., Fuller, J.L., 1965. Genetics and the Social Behaviour of the Dog. University of Chicago Press, Chicago.

Serpell, J.A., Jagoe, J.A., 1995. Early experience and the development of behaviour. In: Serpell, J.A. (Ed.), The Domestic Dog: its Evolution, Behaviour and Interactions with People. Cambridge University Press, UK, pp. 79-102.

Sheppard, G., Mills, D.S., 2003. Evaluation of dog appeasing pheromone as a treatment for fear of fireworks by dogs. Vet. Rec. 152, 432-436.

Slabbert, J.M., Rasa, O.A.E., 1993. The effect of early separation from the mother on pups bonding to humans and pup health. J. S. Afr. Vet. Assoc. 64, 4-8.

Tod, E., Brander, D., Waran, N.K., 2005. Efficacy of dog appeasing pheromone in reducing stress and fear related behaviour in shelter dogs. Appl. Anim. Behav. Sci. 93, 295-308.

Voith, V.L., Borchelt, P.L., 1996. Readings in Companion Animal Behaviour. Veterinary Learning Systems, Trenton, New Jersey.

Wells, D.L., Hepper, P.G., 2000. Prevalence of behaviour problems in dogs purchased from an animal rescue shelter. Appl. Anim. Behav. Sci. 69, 55-65. 